\title{
Sur un cas de Rickettsiose canine à Rickettsia canis, en Oubangui Chari
}

\author{
par J. ITARD
}

La Rickettsiose canine à $R$. canis, découverte en Algerie par Donatien et Lestoquard (1935), fut successivement signalée en France (Montpellier et Marseille) par les mêmes auteurs, au Kenia par Mulligan, en Afrique du Sud par Neitz et Thomas, en Rhodésie par Lawrence, dans le Proche-Orient par Pigoury et Bernard (1939), par Malbrant au Congo (1939), par Mornet au Niger (1940) et Rousselot au Soudan (1942). Elle n'a, jusqu'à présent, ct à notre connaissance, jamais été signalée en Oubangui-Chari.

L'observation que nous relatons concerne une chienne Boxer, âgée de 5 ans, présentée à la consultation du Service de l'Elevage de Bangui le 4 juillet 1957. Cet animal, en excellente santé jusqu'à ce jour, a brusquement perdu l'appétit, est somnolent. Température 390 . Un frottis de sang met en évidence des Piroplasmes ( $B$. canis). Traitement au Zothélone et huile camphrée, Vitascorbol et Hépatrol « $\bar{A}$ ». Deux jours plus tard, la chienne est réexaminée. Etat général sans changement. Un nouvel examen de sang montre quelques rares Piroplasmes. Une nouvelle injection de Zothélone est pratiquée.

Par un heureux hasard, nous revoyons le même jour vers $23 \mathrm{~h}$. cet animal, dont l'état général s'est brusquement aggravé. Température : 40,90. Somnolence. L'animal reste le plus souvent couché, semble très abattu, ne s'alimente presque pas, et, aux dires du propriétaire a déjà maigri. Nous constatons un larmoiement intense avec conjonctivite. L'animal a de la peine à tenir les yeux ouverts, les larmes souillent le museau. Légère dyspnée. Tachycardie. L'ensemble de ces symptômes, et tout particulièrement, la température élevée, le tuphos et le larmoiement, nous font penser à une Rickettsiose. Nous faisons administrer immédiatement une dragée d'auréomycine à $0,25 \mathrm{~g}$, recommandons d'en faire absorber à l'animal une toutes les 3 heures et de nous le présenter dès le lendemain matin à la consultation.

Tie lendemain, la température est tombée à 39,40, l'animal est plus éveillé. Un examen approfondi nous montre :

$1^{\circ}$ une dyspnée très légère,

$2^{0}$ une tachycardie toujours importante,

$3^{\circ}$ une conjonctivite avec larmoiement, en régression,

$4^{0}$ une légère hypertrophie des ganglions préparotidiens,

$5^{0}$ une absence complète d'exanthème.

L'examen du sang périphérique montre :

$1^{\circ}$ Une leucocytose importante, avec nombreux mononucléaires et disparition des éosinophiles,

$2^{\circ}$ des lésions d'anémie traduites essentiellement par de la karyolyse,

$3^{\circ}$ enfin, après de nombreuses recherches, quelques rickettsies dans les monocytes, en morula composée de grains rassemblés en amas.

Le traitement à l'auréomycine est poursuivi, en diminuant les doses à partir du $3 \mathbf{e}$ jour $(3$ dragées à $0,25 \mathrm{~g}$ par jour). Dès le $2^{\mathrm{e}}$ jour la température est revenue à la normale (380). Disparition du tuphos, de la dyspnée. Persistance de la conjonctivite et de la tachycardie combattue à l'aide d'injections quotidiennes de caféine.

La stérilisation parasitaire est très rapidement constatée dans les frottis effectués quotidiennement, ainsi que la réapparition d'éosinophiles. Le traitement à l'auréomycine est cependant poursuivi trois jours durant.

Les symptômes décrits ici diffèrent assez sensiblement de ceux décrits par Malbrant au Congo, 
et semblent rapprocher cette rickettsiose de celle décrite dans le Bassin Méditerranéen. On peut supposer qu'il s'agit d'une rickettsiose "importée ». Cette hypothèse nous paraît peu vraisemblable. Outre que le chien de la précédente observation sejourne depuis plusieurs années, et sans interruption, en Oubangui, la présence reconnue de $R$. canis au Congo en particulier, est en faveur d'une existence réelle de la maladie' en Oubanqui. La symptomatologie incomplète rencontrée ici tient vraisemblablement à ce que nous nous trouvons en début d'infection (très rares rickettsies des monocytes, en morula composée de grains rassemblés signant une dissociation à peine amorcée), et à ce que la thérapeutique instituée de façon massive et précoce a stoppé l'évolution naturelle de l'affection.

Si cette affection n'a jusqu'à présent pas été signalée en Oubangui, il est vraisemblable que cela tient à ce que la piroplasmose, affection concomitante de la rickettsiose, y est extrêmement répandue, et masque les symptômes propres à cette dernière affection. Lorsque, lors d'examens de sang, effectués de façon systématique chez tous les animaux fébriritants présentés à la consultation de la clinique vétérinaire de Bangui, l'existence d'une piroplasmose est mise en évidence, les recherches ne sont pas poussées plus loin, d'autant que les rickettsies des monocytes peuvent être extrêmement rares dans un frottis.

En conclusion, l'existence d'une rickettsiose canine à Rickettsia canis en Oubangui-Chari paraît devoir être confirmée. L'affection se signale essentiellement par une température, élevée, un tuphos profond, du larmoiement. Le traitement à l'auréomycine, institué de façon massive et précoce, donne des bone résultats (baisse de la température dès le $2^{e}$ jour, disparition du tuphos, stérilisation parasitaire).

\section{BIBLIOGRAPHIE}

1. DONATIEN et LESTOQUARD. - Existence en Algérie d'une Rickettsia du chien. $B u l l$. Soc. Path. Exo., (1935), 18, 418.

2. CURASSON. - Traité de Protozoologie Vétérinaire et Comparée. (1943), 3, 380.

3. ROUSSELOT. - Notes de parasitologie tropicale. (1953), 1, 71 .

\section{SUMMARY}

\section{A Case of Rickettsia Canis in Oubangui-Chari, French Equatorial Africa.}

A Boxer bitch, infected with Bigemina canis showed a sudden deterioration of its général condition in spite of its treatment with "zoothelone" and the fact that piroplasms had practically disappeared from its blood. The typhoid syndrome, lacrymation and high temperature as shown by the animal were suspicious of rickettsiosis, and the diagnosis was confirmed by the finding of $R$ : canis in large mononuclears. An early and massive treatment with aureomycin proved very efficient. This is the firts case which was reported in the territory. 